\title{
HR OF THE FUTURE: \\ CONCLUSIONS AND OBSERVATIONS
}

\section{Dave Ulrich}

\section{Introduction}

These 25 essays represent a wonderful cross section of thinking about the future of HR. They are from academics, HR executives, and consultants who spend much of their professional lives thinking about HR issues. Having read the essays, some consensus emerges around several themes:

- HR is under scrutiny, and this scrutiny is a good thing.

- HR as we have known it needs to change.

- Changing HR will represent important challenges and will require new competencies.

- If HR does not meet the challenge of change, it is at risk of being disbanded.

While some answers concerning the future of HR seem to be known and shared; there is more that is unknown. In these cases, the questions are more compelling than answers.

\section{Things Known}

The authors agree to the inevitability of ongoing change. They suggest that the pace and unpredictability of change will increase. Pace means that whatever will happen will happen faster than anticipated. Once the Internet becomes standard, for example, literally millions of people can sign-on overnite to learn new ideas for work and personal lives. What took months to share (e.g., printing and marketing a book, sharing the best practice from within a firm, or publishing research findings) may be disseminated in days or hours. Unpredictability of change means that we can not fully predict what will happen and as pace and unpredictability increase, questions which took a long time to answer need to be answered more quickly. Which organizational forms will become the norm? Which leaders who are deified today will be disparaged tomorrow? Which companies with great reputations today will lose them quickly?

Most essays agree on the drivers for change. Globalization will require seeing and acting beyond local boundaries. Technology will make information more accessible and join people together electronically in ways that can impact organizations and work relationships. A more knowledge-based workforce will make many employees into volunteers because they could choose to work elsewhere for equal or more money, so they work in an organization by choice, not by obligation. Turning worker knowledge into productivity and leveraging intellectual capital will become workforce challenges of the future. Redefining firm performance away from merely cutting cost to profitable growth also will require change.

Knowing the pace and unpredictability of change does not mean that firms have learned to manage change. Change redefines risk. In a low-change world, reducing risk means getting more of the right answer before taking action. In a world of high amounts of change, reducing risk means acting without full answers but having the capacity to adjust mid-stream. Agility becomes more important than accuracy in reducing risk. HR professionals cannot assume that they will design the "perfect" pro- 
gram. They must learn to quickly design thoughtful programs, to act on those programs, then to learn and adjust.

Change comes at two levels. First, fundamental change means changing culture or identity. Firms which have for decades had an identity may find that their traditional culture fails to create current customer value; for example, Sears, IBM, and General Motors are in the midst of fundamental culture or identity change. Fundamental culture change will probably affect almost every firm from government agencies learning to become more responsive and service oriented to universities learning to serve students of all ages and across many geographies to airlines mastering customer loyalty.

Second, capacity for change means responding quickly to that which is occurring around us. Reducing the cycle time for completion of business initiatives becomes the requirement and demand for HR professionals. A firm recently went from concept of an HR competency model to delivery in ten weeks, and the leader of the HR function then asked that a similar process be applied to other staff functions in six weeks. Making things happen more quickly, but still better, is an outcome of change.

From these essays we can conclude that the workplace and workforce of tomorrow will be different from that of today, that change outside HR will require change within HR, and that HR is at a crossroads in its ability to deal with this change.

\section{Things Unknown}

These essays show that we know more about the context for HR in the future than about the content. The content deals will issues of role, focus, practices, and governance of HR in the future. For each of these areas, we see questions which should elicit debate, dialogue, and experimentation over the next few years.

\section{Role of HR: What Is the Future Role of HR?}

A number of continua can be used to describe what the future role of HR might be; each of- fers an array of choices for roles HR can and should play.

- Administrative vs. strategic. The evolution of the function has been from administrative to strategic. There are arguments, however, that if the administrative work is not done efficiently, accurately, and timely, HR professionals cannot plan strategic roles. How to balance and be dualistic in these roles will be an ongoing challenge for the profession.

- HR departments: Existing vs. transformed vs. disappeared. Some argue HR should rediscover its past (paying attention to employee unrest, unions, firm values, and administrative processes). Others argue that HR should be transformed into an elite strategic corps of business partners which creates globally competitive organizations. Others argue that HR departments should disappear and be outsourced. These debates will continue as will the debates as to what to name the HR department (human relations, human resources, employee resources, organizational capability, etc.)

- Doing HR work: HR professional vs. line manager vs. staff-who does HR work? What part of HR work is done by HR professionals, line managers, or other staff groups? In a focus group of line managers talking about the HR department, participants uniformly stated that as HR was becoming "more strategic" most of the traditional HR work fell to them, the line managers and that they did not want to do it. They wanted HR to return to doing HR work (meaning, let them as managers be freed up to manage). How to clearly define roles and accountabilities will be discussed for the next few years.

- Metaphors for HR professionals: leaders, architects, stewards, partners, or players? Image and identity are important because they shape behavior. HR professionals know many of the images they want to shed, e.g., policy police, bureaucrats, administrators, regulators, etc. It is less clear what the future iden- 
tity of HR should be. In all probability, multiple roles will be played by HR professionals depending on the business context and the proclivity of the HR professional, but, knowing alternative metaphors increases the debate.

- Aggressiveness of HR: advocacy vs. acquiescence, proactive vs. reactive? Under what circumstances should HR professionals become more assertive and take a stand? Learning when to have a unique point of view which should be articulated and advocated and when to enlist as part of the team will be ongoing concerns for HR professionals.

Arguments have been well made in these essays for multiple points of view about the future role of HR.

Focus of HR: What Should Be the Focus of HR?

The focus of HR describes more where the work is done. Again, a number of continua highlight questions about the setting for HR.

- Target audience: workforce vs. customer vs. investor vs. government? Investments in HR practices may be focused on improving the workforce (ensuring more competent, committed, and dedicated employees), serving external customers (creating organizational capabilities that customers value and pay for), investors (reducing cost, which leads to profitability), and/or government (crafting policies with national interest). Each audience has merit; balancing the needs of multiple audiences raises questions about the focus of HR.

- HR work: domestic vs. global? Globalization has moved from a buzzword to a reality; however, crafting HR work so that local organization needs are served along with global requirements will force rethinking many HR tasks.

- HR constituents: within firm vs. across alliances? A number of the essays point out that future HR work will be housed in organizations that differ greatly from what we have today. As organizations be- come more an assortment of alliances and relationships, HR practices will also need to cross boundaries. Legal definitions of firm boundaries cannot be constraints for HR practices. We need to learn how to leverage HR work both within the firm and across firm alliances.

The setting for HR will change in response to new organizational arrangements and agreements. These settings will require more flexible, dynamic, and responsive HR professionals.

Practices of HR: What Are the Emerging HR Practices?

The essays point to a number of emerging HR practice areas. They are areas that will require investments of time, talent, and resources to turn a set of ideas into tools. Some of these areas include:

- Building leadership bench: What are the competencies for the leader of the future, both at the top and the middle of the organization? How can HR practices be crafted to develop leaders who meet tomorrow's needs today? These essays contain wonderful examples of how leaders will need to lead in the future and what the subsequent HR implications are supporting the development of such leaders.

- Creating organizational capabilities (knowledge based organization/high capability organization): Organizations often take on lives of their own characterized by personalities, traits, and habits. HR professionals will need to learn how to codify and create aligned organizational capabilities in addition to individual competencies by addressing such questions as: What are critical organizational capabilities? How are they created? How are they changed?

- Enhancing knowledge transfer: Knowledge transfer means that best practices are shared within a firm, among firms, and between firms and government. HR professionals must master tools of learn- 
ing such as knowledge generation (e.g., experimentation, benchmarking, continuous improvement, competence acquisition) and knowledge generalization (e.g., moving information, skills, decision making, and rewards across boundaries). As knowledge-based organizations proliferate, HR professionals play different roles.

- Leveraging technology: Technology will change how work is done in general and how HR is practiced in particular. A sample of HR-related technology questions include: How will technology connect employees without face-to-face contact? How will technology change communication patterns (e.g., electronic all-hands meetings)? How will technology change specific HR practices (e.g., resumes through Internet, distance learning for training, automated performance reviews, tailored benefit programs)?

The traditional HR practices of staffing, training, performance management, benefits, regulation, labor relations, and so forth will not go away, but they will become the table stakes for HR, with new practices emerging constantly.

\section{Governance of HR: How Do We Get HR Work Done?}

The practical act of doing HR work will change. Several themes emerge from these essays as to how HR work will be governed.

- Deliverables more than doables: For decades HR has focused on doing good work through design of programs that affect people and processes. Increasingly, the emphasis must be more on deliverables. Deliverables represent the results or outcomes of doing good HR work. What happens because we have crafted innovative staffing, training, or high-performing team programs? What are the organizational implications? These essays point to a number of possible deliverables: making employees volunteers, implementing strategy, cre- ating economic value, ensuring cultural heritage, managing employee work/life needs, globalization, etc.

- Benchmarking: Institutionalization theory reviews the process of sharing ideas across boundaries. In the HR world, this has been operationalized as benchmarking and best practices. Conferences, publications, consultants, and other forums exist where ideas are quickly shared from one unit to another. HR professionals must become masters of benchmarking by not falling into the "if they did it, so must we" trap.

- Measuring more and more accurately: Too often, HR works at the personal whim of a CEO. When the CEO "takes a liking" to HR issues, HR gets attention; when she or he does not, it does not. These essays call for and predict a more rigorous and demanding measurement process for HR in the future. How do we know when HR works? How do we tie HR work to business results? In the next few years, we will see more precise, valid, and reliable measures of HR effectiveness.

- Theory based vs. haphazard: From their roots in these essays a wonderful mosaic of theories of HR emerges. Some of these theories focus on individual development (e.g., HR's role in developing leaders and nurturing employee well-being). Some theories focus on organizations (e.g., HR's role in coordinating work across flexible and alliance organizations).

- Change and continuity: In the midst of predicting a dramatically different future for HR, some of the essays thoughtfully demonstrate that much of what HR has done, it must continue to do. Employees have always been hired, developed, and paid; and organizations have always had to have processes to take care of employees. Much of the past will be found in the future, but learning what of the past to keep and what to change may be an ongoing governance issue.

At minimum, some HR work will be done differently. If an HR professional from a previous 
decade arrives in the year 2000, expectations, skills, and outcomes will be different.

\section{Final Thoughts}

If our purpose is to propose a debate about the future, it is better to end with questions than with answers. Questions elicit new frameworks, approaches, and alternatives, so, the fi- nal two questions we would ask (with our answer) are:

Do you want to play in this always changing and at times unclear future?

Are you having fun?

Without a doubt, all the fine authors in this issue and many others of the best HR professionals we know answer with a resounding "yes." 